\title{
ROTATIONAL ASYMMETRIC MULTIFOCAL INTRAOCULAR LENS - USE IN PATIENT UNDERGOING CATARACT SURGERY
}

\author{
Ziak $\mathrm{P}^{1,2}$, Halicka ${ }^{1,2}$, Mojzis $\mathrm{P}^{3}$, Kralik $\mathrm{M}^{2}$,Nikel J. ${ }^{2}$ \\ ${ }^{1}$ Comenius University, Jessenius Faculty of Medicine in Martin and University \\ Hospital, Martin, Slovakia, Clinic of Ophtalmology; ${ }^{2}$ UVEA MEDIKLINIK, s.r.o., Martin; \\ ${ }^{3}$ Premium Clinic, Teplice, Czech Republic
}

\begin{abstract}
Introduction: A cataract is a clouding of the lens in the eye leading to a decrease in vision. Cataracts are the cause of half of blindness and 33\% of visual impairment worldwide. Surgery with phacoemulsification followed by implantation of intraocular lens (IOL) is gold standard treatment for cataract. In some cases multifocal IOLs are used. This is the first published use of rotational asymmetric multifocal IOL in cataract surgery in Slovakia.

Method: In the study 78 eyes of 58 patients (mean age 62.3 years) were implanted for a cataract with the rotational asymmetric multifocal IOL. Corrected and uncorrected distance and near visual acuities were analysed preoperatively, 1 month and 6 months postoperatively. Contrast sensitivity was tested at 6 months postoperatively. The Friedman Two -Way Analysis of Variance and Multiple Comparison Test were used for all parameter comparisons, in all cases, the same level of statistical significance $(p<0.05)$ was considered significant.

Results: Average preoperative uncorrected distance visual acuity has increased from 0.32 to 0.87 and 0.93 respectively in 1 month and 6 months after surgery $(\mathrm{p}<0.05, \mathrm{n}=78)$. Average preoperative uncorrected near visual acuity (UCNVA) has increased from 0.2 to 0.77 and 0.79 respectively in 1 month and 6 months after surgery (p $<0.05$ ). Average preoperative best corrected distance visual acuity has increased from 0.65 to 0.95 and 1.03 respectively in 1 month and 6 months after surgery $(\mathrm{p}<0.05)$. Significant improvement was found also between preoperative best corrected near visual acuity and UCNVA 1 and 6 month after surgery $(p<0.05)$.

In conclusion, the rotational asymmetric multifocal IOL LS-313 MF 30 provides good visual rehabilitation for near and distance vision after cataract surgery. Contrast sensitivity outcomes account for the patient satisfaction with this innovative IOL technology.
\end{abstract}

Key Words: cataract, multifocal, intraocular lens, rotational asymmetric

\section{INTRODUCTION}

A cataract is a clouding of the lens in the eye leading to a decrease in vision. It can affect one or both eyes. Often it develops slowly and are rarely dangerous, after a number of years they will likely affect vision. Symptoms include decrease in clarity of vision, that is not correctable with glasses, faded or washed out colors, sensitivity to light glare and halos around lights and frequent changes to eyeglass prescription (1). Cataracts are the cause of half of blindness and $33 \%$ of visual impairment worldwide (2).

Cataracts are most commonly due to aging, but may also occur due to trauma, radiation exposure, be present from birth, or occur following eye surgery for other problems $(3,4)$. Risk factors include diabetes, smoking tobacco, prolonged exposure to sunlight, and alcohol (5). Due to metabolic changes of the crystalline lens fibers are deposited clumps of protein or yellow-brown pigment in the lens reducing the transparency. Diagnosis is mainly done by an slit lamp eye examination (1).

Surgery is only needed if the cataract is causing subjective problems. During surgery is cloudy natural lens removed and replaced with an artificial lens. Usually in cataract surgery is used mono-focal intraocular lens (IOL) with postoperative refraction setup for either good distance or near vision. In some cases, mostly in hyperopic patients, who want to see

Address for correspondence:

MUDr. Žiak Peter, PhD., Clinic of Ophtalmology, Jessenius Faculty of Medicine in Martin, Comenius University, Kollarova Str. N. 2, 03659 Martin, Slovak Republic.

e-mail:ocnesekr@unm.sk 
distance and also read near texts without using correction glasses multifocal IOLs are used. Here we present our results of first use of rotational asymmetric multifocal IOL in cataract surgery in Slovakia.

\section{METHODS}

This retrospective study enrolled patient with cataract who had phacoemulsification followed by implantation of multifocal IOL between January 2013 and April 2015. The rotational asymmetric multifocal IOL with a refractive design, combining an aspheric distance vision zone with a sector-shaped near vision zone with a $+3.00 \mathrm{D}$ add (Oculentis, Germany) was used in all cases. This foldable IOL has an overall length of $12.0 \mathrm{~mm}$, an optic diameter of $6.0 \mathrm{~mm}$, and a plate-haptic design. The IOL is of a hydrophilic acrylic material with a hydrophobic surface.

In the study 78 eyes were implanted for a cataract. The mean age was 62.3 years at the time of surgery, with a range of $45-84$ years. Of the 58 patients, 35 were female. Scotopic pupil diameter average was $4.6 \pm 1.06 \mathrm{~mm}$ and photopic $3.12 \pm 0.64 \mathrm{~mm}$. A comparison was made between the sphere and the astigmatic components before and after surgery. All patients provided informed consent. Exclusion criteria were a history of glaucoma or retinal detachment (RD), corneal disease, corneal surgery, ocular inflammation, macular degeneration, or retinopathy.

Preoperative biometry was performed with optical biometry system IOLmaster (Carl Zeiss Meditech, Oberkochen, Germany). The Hoffer- $Q$ formulae were used in the IOL calculation. All patients were implanted with the LS-313 MF30 lens (Oculentis) during standard phacoemulsification surgery. At 1 and 6 months after surgery was performed ophthalmic examination - refraction measurement (Nidek, Japan), uncorrected and corrected distance and near visual acuity (Snellen charts), contrast sensitivity (LCD optotype CX-1000, Topcon, Japan) and slit-lamp examination.

The procedures were performed at 2 surgical centers (Eye clinic in University Hospital in Martin and UVEA Mediklinik, Martin) by 2 experienced surgeons. A local anesthesia was given in all cases, and the patient was prepared and draped for surgery. Most incisions were made on the steepest corneal meridian to neutralize corneal astigmatism. After phacoemulsification, the IOL was inserted in the capsular bag through a $2.75 \mathrm{~mm}$ corneal incision using a Viscoject 2.2 injector (Cartridge-Set LP604240M, Oculentis GmbH) with the reading add placed inferiorly. Surgery in the second eye was usually performed 1 week later.

Postoperatively, patients were instructed to instill 1 drop of levofloxacin $0.5 \%$ (Oftaquix) 5 times daily for 1 week and 1 drop of loteprednol 0.1\% (Lotemax) 5 times daily for 1 month, with decreasing dosage each week of 1 drop.

Statistical analysis: Statistical analysis was performed using SYSTAT (Systat Software, San Jose, CA). The Friedman Two-Way Analysis of Variance and Multiple Comparison Test were used for all parameters comparisons between preoperative and postoperative examinations as well as between consecutive postoperative visits. In all cases, the same level of statistical significance $(\mathrm{p}<0.05)$ was considered significant.

The main outcomes measured were as follows: uncorrected and best corrected distance and near visual acuities and contrast sensitivity.

\section{RESULTS}

Pre-operational topography was measered average cylinder $-0.98(43.11 \mathrm{x} 43.99)$ and 1 month post-op -0.64 (43.01 x 43.70) to 6 months - 1.06 (43.10 x 43.98). Pre-op refraction average was -0.24Dsf and -0.14Dcyl, 1 month after -0.07Dsf and -0.13Dcyl and 6 months after-0.03Dsf and -0.11Dcyl. 
A significant improvement was observed postoperatively in uncorrected distal visual acuity (UCDVA), best corrected distal visual acuity (BCDVA) and uncorrected near visual acuity (UCNVA). Average preoperative UCDVA has increased from $0.32 \pm 0.2$ to $0.87 \pm 0.2$ and $0.93 \pm 0.26$ respectively in 1 month and 6 months after surgery $(\mathrm{p}<0.05, \mathrm{n}=78)$, see figure 1, part a. Average preoperative BCDVA has increased from $0.65 \pm 0.28$ to 0.95 \pm 0.18 and $1.03 \pm 0.21$ respectively in 1 month and 6 months after surgery $(\mathrm{p}<0.05)$, see Fig. 1, part b. Average preoperative UCNVA has increased from $0.2 \pm 0.27$ to $0.77 \pm 0.18$ and $0.79 \pm 0.19$ respectively in 1 month and 6 months after surgery $(p<0.05)$, see Fig. 1, part c. Significant improvement was found also between preoperative BCDVA and UCDVA 1 and 6 month after surgery: $0.65 \pm 0.28$ vs. $0.87 \pm 0.2$ and $0.93 \pm 0.26$ respectively ( $\mathrm{p}<0.05)$, see Fig. 2, part e. No significant change was found between BCNVA preoperatively and UCNVA postoperatively; $0.81 \pm 0.21$ vs. $0.77 \pm 0.18$ and $0.79 \pm 0.19$ (p > 0.05), see Fig. 2, part d.

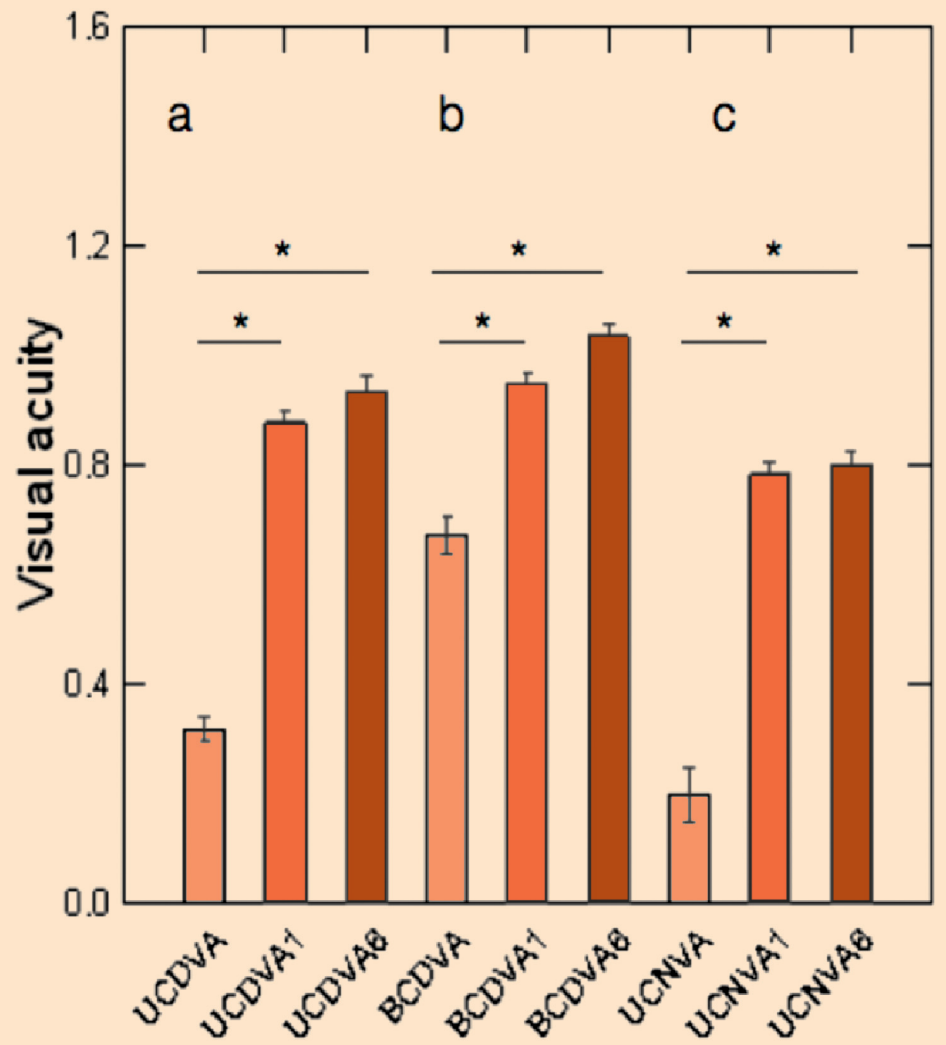

Fig. 1: Visual acuity results: a) plot of uncorrected distal visual acuity before, 1 month and 6 months after surgery, significant results were recorded 1 and 6 months after surgery $(p<0.05)$, b) plot of best corrected distal visual acuity before, 1 month and 6 months after surgery, significant results were recorded 1 and 6 months after surgery $(\mathrm{p}<0.05)$ and part c) plot of uncorrected near visual acuity before, 1 month and 6 months after surgery, significant results were recorded 1 and 6 months after surgery $(\mathrm{p}<0.05)$ 


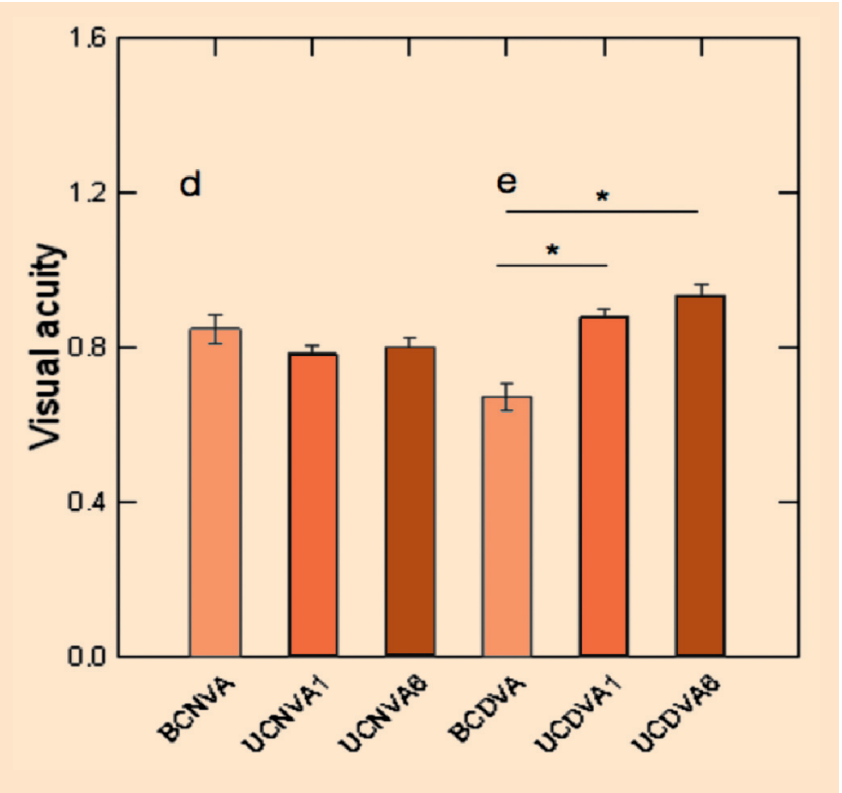

Fig. 2. Visual acuity change as effect of cataract surgery: d) plot of best corrected near visual acuity vs. uncorrected near visual acuity 1 month and 6 months after surgery, no significant results were found ( $p>0.05)$, e) plot of best corrected distal visual acuity before vs. uncorrected distal visual acuity 1 month and 6 months after surgery, significant results were recorded 1 and 6 months after surgery $(\mathrm{p}<0.05)$

Contrast sensitivity (CS), tested with spatial frequency graphs, was found to be $95.75 \%$ from maximum contrast sensitivity for 3 cycles per degree ; $81.95 \%$ for 6 cycles per degree; $67.1 \%$ for 12 cycles per degree and $61 \%$ for 18 cycles per degree (see Fig. 3).

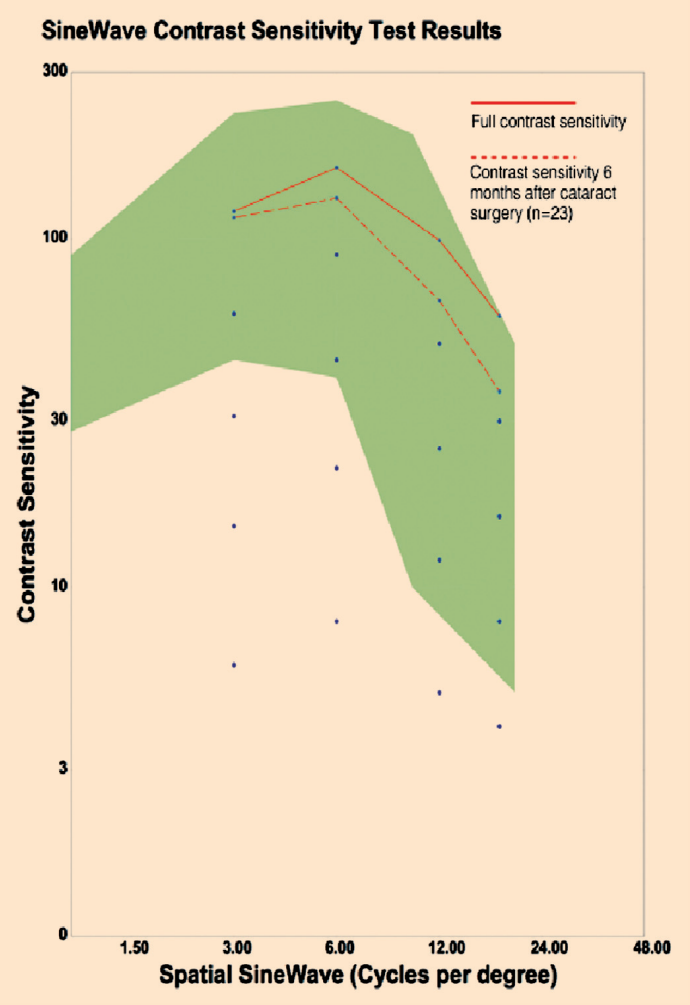

Fig. 3 Contrast sensitivity measured 6 months after cataract surgery in group of patients. 


\section{DISCUSSION}

The goal of cataract surgery is to provide complete visual rehabilitation to patients with the surgery. With the improvement of IOL technology such as multifocal IOLs, patients have achieved acceptable visual acuity for distance and near vision, with a slight limitation for intermediate vision.

Visual and refractive outcomes were evaluated. As expected, a significant improvement in distance and near visual acuity was obtained. These outcomes in distance and near vision were consistent with previous studies with multifocal IOLs (6). Visual outcomes obtained with this model of IOL for all distances are similar to those achieved with different IOL designs, e.g. diffractive multifocal IOL (7). As a result of cataract also BCDVA before surgery was significantly lower to UCDVA postoperatively. Surgery therefore had effect on both parameters of UCDVA - transparency of the optic media and postoperative refraction close to zero. There was no significant change in BCNVA pre-operatively vs. UCNVA postoperatively, this finding have to possible explanation - magnification of glasses prescription in presbyopia and design of rotational asymmetrical multifocal IOL.

Contrast sensitivity obtained with this IOL model were within or near the normal limits for the age sample analyzed. These outcomes were consistent with previous studies with other multifocal IOLs (8). Limitation is that CS was not performed before cataract surgery as it was shown in another studies (9).

One important issue in assessing the success of the surgery relates to selection of patients who are in good condition to receive intraocular implantation of multifocal lenses. Intraocular lenses that can give clear vision at all distances with few adverse effects are still being developed. Patients should be informed about the possible limitations and unwanted effects, such as the presence of haloes around lights, photopsia and difficulties regarding intermediate vision. Even though the majority of these unwanted symptoms generally disappear with neuroadaptation, the success of this procedure depends on the patient's understanding of these possible problems and on his or her expectations.

In conclusion, the LS-313 MF 30 rotational asymmetric multifocal IOL provides good visual rehabilitation for near and distance vision after cataract surgery. Contrast sensitivity outcomes account for the patient satisfaction with this innovative IOL technology.

\section{REFERENCES}

1. Allen D, Vasavada A. Cataract and surgery for cataract. BMJ 2006; 333 (7559): 128-32.

2. WHO.Visual impairment and blindness Fact Sheet $\mathrm{N}^{\circ} 282$. August 2014.

3. Worgul BV, Merriam GR Jr, Medvedovsky C. Cortical cataract development-an expression of primary damage to the lens epithelium. Lens Eye Toxic Res. 1989;6(4):559-71. Review.

4. Truscott RJ, Friedrich MG. The etiology of human age-related cataract. Proteins don't last forever. Biochim Biophys Acta. 2016 Jan;1860(1 Pt B):192-8.

5. Mamatha BS, Nidhi B, Padmaprabhu CA, Pallavi P, Vallikannan B. Risk Factors for Nuclear and Cortical Cataracts: A Hospital Based Study. J Ophthalmic Vis Res. 2015 Jul-Sep;10(3):243-9.

6. Vingolo EM, Carnevale C, Fragiotta S, Rigoni E, Iacobelli L. Visual Outcomes and Contrast Sensitivity after Bilateral Implantation of Multifocal Intraocular Lenses with +2.50 or +3.0 Diopter Addition: 12-Month Follow-Up. Semin Ophthalmol. 2016 Apr 29:0.

7. Venter JA, Pelouskova M, Bull CE, Schallhorn SC, Hannan SJ. Visual outcomes and patient satisfaction with a rotational asymmetric refractive intraocular lens for emmetropic presbyopia. J Cataract Refract Surg. 2015 Mar;41(3):585-93.

8. Mojzis P, Pena-Garcia P, Liehneova I, Ziak P, Alio JL. Outcomes of a new diffractive trifocal intraocular lens. J Cataract Refract Surg 2014; 40:60-69

9. Stifter E, Sacu S, Thaler A, Weghaupt H. Contrast acuity in cataracts of different morphology and association to self-reported visual function. Invest Ophthalmol Vis Sci. 2006;47:5412-5422.

Received: July,1,2016

Accepted: August,20,2016 